Ann. Biol. anim. Bioch. Biophys., I969, 9 (4), 537-553.

\title{
LES PRINCIPALES VOIES DU MÉTABOLISME CALCIQUE CHEZ LE PORC EN CROISSANCE
}

\author{
P. BESANÇON et L. GUÉGUEN \\ avec la collaboration technique de Colette Katz et J. GaUthIER \\ Station centrale de Recherches de Nutrition, \\ Centre national de Recherches zootechniques, 78 -Jouy-en-Josas \\ Institut national de la Recherche agronomique
}

SOMMAIRE

Les principaux paramètres du métabolisme du calcium ont été mesurés chez ro porcs en croissance, pesant de 30 à $4^{\circ} \mathrm{kg}$, après une injection unique intraveineuse de 2 à $3 \mathrm{mCi} \mathrm{de}{ }^{45} \mathrm{Ca}$.

Des bilans calciques ont été effectués, les pertes endogènes fécales étant mesurées, soit par la méthode classique de dilution isotopique, soit à l'aide des courbes d'excrétion fécale cumulée de radioactivité. L'interprétation des courbes de décroissance de l'activité spécifique du calcium sanguin a permis de déterminer les paramètres osseux à l'aide de la méthode de BAUER et al. et de la méthode de Aubert et Mirhaud. Après confrontation et discussion des deux méthodes, un modèle simplifié à trois compartiments a été choisi. Les limites de validité de la méthode de BAUER ont été définies; cette méthode conduit à une surestimation systématique de la vitesse d'incorporation du calcium dans l'os.

Le coefficient d'utilisation digestive réelle du calcium alimentaire est de $45 \mathrm{p}$. roo. Les pertes fécales de calcium endogène représentent $77 \mathrm{p}$. Ioo des pertes fécales totales de calcium, tandis que l'excrétion urinaire est négligeable.

Les animaux, dont le gain de poids journalier est de $300 \mathrm{à} 600 \mathrm{~g}$, retiennent $3,4 \mathrm{~g}$ de calcium par jour, soit 3I p. Ioo du calcium ingéré. L'accrétion totale de calcium, qui mesure l'anabolisme osseux, s'élève à 10,7 $\mathrm{g}$ par jour, et la résorption (catabolisme osseux) est de $7,3 \mathrm{~g}$ de calcium par jour. Près de la moitié de l'accrétion mesurée, soit $45 \mathrm{p}$. roo, est d'origine endogène, par suite du recyclage interne du calcium osseux. Il est ainsi possible de mesurer l'aptitude de l'os à se renouveler.

La masse de calcium du pool échangeable a été estimée à $24 \mathrm{~g}$ en moyenne.

Par l'intensité de son métabolisme minéral osseux, le Porc se rapproche davantage du Rat que de l'Homme.

\section{INTRODUCTION}

L'os, comme les autres tissus, est soumis à un continuel renouvellement et les aspects dynamiques du métabolisme minéral osseux sont déjà bien connus à la suite de nombreux travaux. L' " accrétion " est le phénomène par lequel le calcium, comme les autres éléments minéraux ostéotropes, est incorporé dans l'édifice cristallin des 
tissus calcifiés (BAUER et al., I955 $a$, I96I). On a également montré que le calcium est en partie soumis à des phénomènes d'échanges (FALKENHEIM et al., I95I ; DALIEMAGNE et al., I955; NEUMAN et al., I958; TRIFFI'T et al., I968). Une fraction du calcium de l'organisme constitue un " pool " échangeable, lui-même subdivisé en plusieurs compartiments (BAUER et al., I955 $a$ et $b$, AUBERT et MILHAUd, Ig6o).

Il a paru intéressant, dans certains cas, de chercher à définir les relations qui existent entre les paramètres du métabolisme calcique d'une part, et la vitesse de croissance ou l'âge de l'animal d'autre part. Chez le Rat, par exemple, la diminution de la rétention calcique avec l'âge est non seulement due à une moins bonne absorption intestinale, mais aussi, au niveau de l'os, à une réduction de l'anabolisme osseux et de l'accrétion du calcium (Michavo et al., I963; LEMaIRE, I967 ; BoHR, I968). Par ailleurs, d'après COHEN et STOCLET (I962), les échanges calciques sont d'autant plus intenses que la vitesse de croissance de l'animal est plus forte.

Le Porc, qui manifeste une vitesse de croissance élevée, puisqu'elle peut représenter une augmentation journalière de près de $2 \mathrm{p}$. Ioo du poids vif, a été peu étudié dans ce domaine. Des travaux de HANSARD et al. (I96r) ont montré les variations de l'utilisation et de la rétention du calcium avec 1'âge chez le Porc, mais les résultats ne permettent pas de connaître les étapes intermédiaires et, en particulier, celles du métabolisme osseux. Cette étude se propose de décrire les principales voies du métabolisme calcique chez le Porc en croissance par l'emploi du calcium-45 associé à des mesures de bilans classiques.

\section{I. - MATÉRIEL E'T MÉTHODES}

\section{Animaux et protocole expérimental}

Les essais ont porté sur dix porcs mâles en croissance, de race Large White, pesant de 30 à

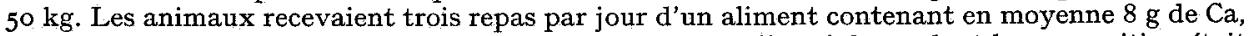
$7,5 \mathrm{~g}$ de $\mathrm{P}$ total et $\mathrm{I}$ ooo UI de vitamine $\mathrm{D}_{3}$ par $\mathrm{kg}$ de matière sèche et dont la composition était la suivante :

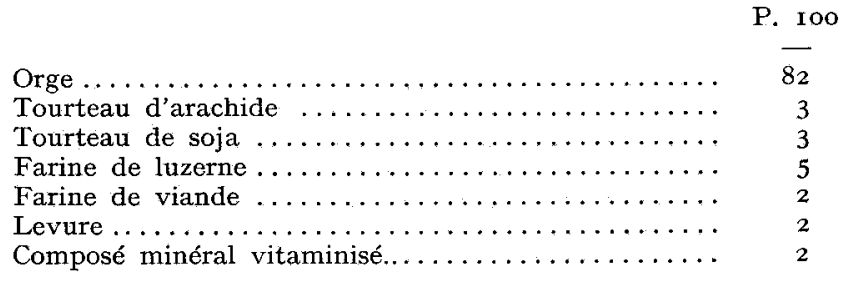

Après avoir été munis de cathéters permanents dans la veine jugulaire externe en vue de faciliter les injections intraveineuses et les prélèvements de sang, les animaux ont été mis en cages à métabolisme. Après une période d'adaptation de quelques jours, les bilans calciques ont été mesurés durant une dizaine de jours. En outre, une injection unique de calcium-45 a été faite par voie intraveineuse sous forme de $\mathrm{CaCl}_{2}$ injectable en solution isotonique. La quantité d'élément calcium injectée a été systématiquement inférieure à $20 \mathrm{mg}$ pour une activité de $2 \mathrm{mCi}$ environ. Après cette injection de radiocalcium, des prélèvements de sang ont été effectués plusieurs fois le premier jour, puis en moyenne une fois par jour jusque vers 200 ou 300 heures. Les urines et les fèces ont été recueillies en totalité durant au moins dix jours, d'abord trois fois par 24 heures, puis une seule fois à partir du $4^{\mathrm{e}}$ jour. 


\section{Dosages et mesures}

Tous les échantillons de sang, d'urine et de fèces ont été minéralisés par voie sèche au four à $55^{\circ} \mathrm{C}$; les cendres ont été reprises par $\mathrm{HCl}$ concentré à chaud puis par $\mathrm{HNO}_{3}$ à Io p. Ioo et diluées. Le calcium a été dosé par photométrie d'émission de flamme (GutGuen et Rombauts, r96r) et le phosphore par colorimétrie du phosphovanadomolybdate d'ammonium.

La radioactivité a été mesurée sur les mêmes solutions minéralisées, soit au moyen d'un compteur Geiger-Müller à fenêtre mince et à flux gazeux d'hélium, soit par scintillation liquide. Les comptages effectués avec le compteur GM ont été faits sur I $\mathrm{ml}$ de solution séché sous infrarouge. Les erreurs d'autoabsorption du rayonnement de ${ }^{45} \mathrm{Ca}$ (énergie : $0,25 \mathrm{MeV}$ ) ont été systématiquement corrigées. Les mesures par scintillation liquide ont été faites suivant une technique proposée par PATterson et GREene (1965), le mélange scintillant étant composé de deux parties de toluène-PPO-POPOP (1), à raison de $4 \mathrm{~g}$ de PPO et de $100 \mathrm{mg}$ de POPOP par litre de toluène et d'une partie de Triton X Ioo; on utilise $\mathrm{I} 7 \mathrm{ml}$ de ce scintillateur pour $5 \mathrm{ml}$ de solution.

\section{Calcul de l'excrétion fécale de calcium endogène}

La méthode de dilution isotopique a été décrite par LOFGREEN et KLEIBER (I954, I960) pour mesurer l'excrétion fécale de phosphore endogène. Elle peut être transposée au cas du calcium. En effet, après une injection unique de ${ }^{45} \mathrm{Ca}$ par voie intraveineuse, la radioactivité retrouvée dans les fèces provient uniquement du calcium endogène. Ainsi, au bout d'une huitaine de jours, on peut admettre que l'activité spécifique du calcium endogène fécal est la même que celle du calcium sanguin et on peut écrire la relation:

$$
\mathrm{S} \times \mathrm{F}_{e}=\mathrm{S}_{f} \times \mathrm{F}
$$

où $\mathrm{S}$ est l'activité spécifique du Ca sanguin, $\mathrm{S}_{f}$ l'activité spécifique du Ca fécal total, $\mathrm{F}$ l'excrétion fécale totale de $\mathrm{Ca}, \mathrm{F}_{e}$ l'excrétion fécale endogène de $\mathrm{Ca}$, que l'on peut calculer à partir des autres données déterminées expérimentalement. Il est indispensable de tenir compte de la vitesse du transit digestif qui introduit un décalage d'environ 2 jours entre les activités spécifiques du sang et des fèces.

Une autre méthode est basée sur le fait qu'après une injection intraveineuse de ${ }^{45} \mathrm{Ca}$, la fraction de la dose de radioactivité excrétée par voie fécale au temps infini (valeur obtenue à partir des courbes d'excrétion cumulée, fig. I) est proportionnelle à la vitesse d'excrétion endogène fécale du calcium et à l'intégrale des activités spécifiques du calcium sanguin :

$$
f_{e}=\mathrm{F}_{*} \int_{0}^{\infty} \mathrm{S}(t) d t
$$

où $f_{e}$ est la fraction de la dose de radioactivité éliminée par voie endogène fécale, au temps infini, $\mathrm{S}(t)$ l'activité spécifique du calcium sanguin, $\mathrm{F}_{e}$ la valeur recherchée de l'excrétion endogène fécale de Ca.

L'accord entre les résultats obtenus par ces deux méthodes est excellent.

\section{Méthodes de mesure des principaux paramètres du métabolisme calcique}

Les méthodes qui permettent d'estimer les paramètres du métabolisme calcique sont basées sur la combinaison des résultats de bilans et l'interprétation des courbes de décroissance d'activité spécifique du calcium sanguin après l'injection intraveineuse de radiocalcium.

a) Méthode de BAUER et al., (1965 a, 1961).

Après l'injection unique de ${ }^{45} \mathrm{Ca}$ dans le sang, la radioactivité se répartit sous deux formes : d'une part, elle est diluée dans le pool de calcium échangeable et, d'autre part, elle est incorporée dans la phase minérale osseuse par accrétion. Si l'on admet que l'activité spécifique du calcium sanguin est la même que celle du calcium du pool échangeable, on peut écrire la relation :

$$
\mathrm{R}(t)=\mathrm{E} \mathrm{S}(t)+\mathrm{A} \int_{0}^{\mathrm{t}} \mathrm{S}(t) d t
$$

(1) PPO : 2,5-diphényloxazole ; POPOP : I,4-bis-2-(5-phenyloxazolyl)-benzène. 
où $\mathrm{R}(t)$ est la fraction de la dose de radioactivité retenue au temps $t, \mathrm{~S}(t)$ l'activité spécifique du calcium sanguin au temps $t$, E la masse de calcium échangeable du pool, A la vitesse d'incorporation de $\mathrm{Ca}$ dans l'os ou accrétion.

Connaissant expérimentalement $\mathrm{R}(t), \mathrm{S}(t)$ et donc $\int_{0}^{t} \mathrm{~S}(t) \mathrm{d} t$, il suffit de résoudre cette équation à deux instants $t_{1}$ et $t_{2}$ pour déterminer les valeurs du pool $\mathrm{E}$ et de l'accrétion $\mathrm{A}$.

La méthode nécessite de connaître les valeurs de la rétention de la dose de radioactivité à deux instants $t_{1}$ et $t_{2}$; il faut considérer comme non retenu ce qui a été sécrété dans l'intestin mais qui n'est pas encore excrété dans les fèces. Ainsi, les valeurs ‘ُe la rétention mesurées expérimentalement à partir des valeurs de radioactivité cumulées retrouvées dans les urines et les fèces sont entachées d'erreurs. L'erreur est d'autant moins importante que le temps écoulé depuis l'injection est plus grand. On peut écrire la relation :

$$
u+f_{e}=\left(\mathrm{U}+\mathrm{F}_{e}\right) \int_{0}^{\infty} \mathrm{S}(t) \mathrm{d} t
$$

où $u$ et $f_{e}$ sont les fractions de la dose de radioactivité éliminées respectivement par voie urinaire et fécale, au temps infini, $U$ et $F_{e}$ les valeurs des excrétions endogènes de calcium par voie urinaire et fécale, $\mathbf{S}(t)$ l'activité spécifique du calcium sanguin.

En supposant qu'au temps infini les valeurs de $\left(u+f_{e}\right)$ mesurées expérimentalement sont justes, il est possible de recalculer à partir de cette relation les valeurs antérieures de l'excrétion $\left(u+f_{e}\right)$ et de corriger ainsi les valeurs de la rétention de radioactivité. Pratiquement, l'excrétion de radioactivité devenant négligeable après 200 heures, il n'est pas nécessaire d'attendre plus longtemps (fig. I).

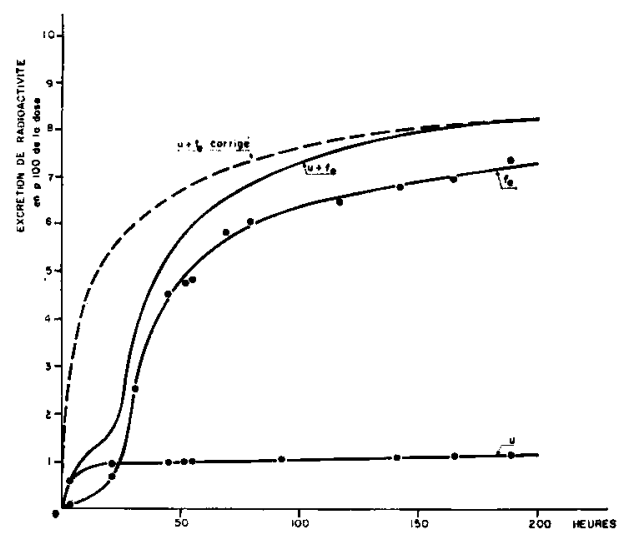

FIG. I. - Excrétion cumulée de radioactivité dans les urines et les fèces

\section{b) Méthode de Aubert et Mirhaun (1960).}

Après 1 'injection intraveineuse de ${ }^{45} \mathrm{Ca}$, la courbe de décroissance de l'activité spécifique du calcium sanguin peut se décomposer en autant d'exponentielles qu'il y a de compartiments de calcium plus ou moins rapidement échangeables. L'ensemble de ces compartiments $\mathbf{M}_{1}, \mathbf{M}_{2}, \mathbf{M}_{\mathbf{3}}$, .. constitue le "fonds commun " ou pool échangeable. La forme générale de l'équation de la courbe de décroissance est une somme de fonctions exponentielles :

$$
\mathrm{S}(t)=\mathrm{A}_{1} \mathrm{e}^{-a_{1} t}+\mathrm{A}_{2} \mathrm{e}^{-a_{2} t}+\mathrm{A}_{3} \mathrm{e}^{-a_{3} t}+\cdots
$$

On peut résoudre le système complet par la méthode proposée par AuBerT et MILHaud (I960). Nous nous sommes limités, dans le cas d'un modèle à trois compartiments, au calcul de la masse de calcium du compartiment le plus rapidement échangeable $\mathrm{M}_{1}$ et de la vitesse de sortie de $\mathrm{Ca}$ hors du fonds commun, somme de l'anabolisme osseux ou accrétion et des excrétions endogènes fécale et urinaire. On peut écrire :

$$
\mathrm{R}_{1}=\mathrm{V}_{\mathrm{T}} \int_{0}^{\infty} \mathrm{S}(t) \mathrm{d} t
$$


où $R_{i}$ est la dose de radioactivité injectée, $\mathrm{S}(t)$ l'activité spécifique du calcium sanguin au temps $t$, $V_{T}$ la vitesse totale de sortie de Ca hors du fonds commun.

On en déduit :

$$
V_{T}=\frac{R_{1}}{\frac{A_{1}}{a_{1}}+\frac{A_{2}}{a_{2}}+\frac{A_{3}}{a_{3}}}
$$

Sachant par ailleurs qu'au temps initial les compartiments $M_{2}$ et $M_{3}$ ne sont pas marqués par le calcium-45 et que l'activité spécifique au temps zéro s'écrit $S(o)=A_{1}+A_{2}+A_{3}$, on en déduit que la masse de $\mathrm{Ca}$ dans le premier compartiment est donnée par:

$$
\mathrm{M}_{1}=\frac{\mathrm{R}_{i}}{\mathrm{~A}_{1}+\mathbf{A}_{\mathbf{2}}+\mathbf{A}_{3}}
$$

Si l'on se limite à un modèle très simplifié à deux compartiments, il est aisé de calculer la masse de calcium $\mathrm{M}_{2}$ dans le $2^{\mathrm{e}}$ compartiment ainsi que la vitesse d'échange $V_{1}$ entre $M_{1}$ et $M_{2}$. Nous verrons au cours de la discussion des résultats que ce modèle simplifié à deux compartiments ne donne pas de résultats très satisfaisants.

\section{II. 一 RÉSULTATS}

Malgré la grande variabilité individuelle qui ressort de l'examen des résultats, il est possible de retenir des valeurs moyennes afn de dégager les principales caractéristiques du métabolisme calcique chez le Porc en croissance (fig. 2).

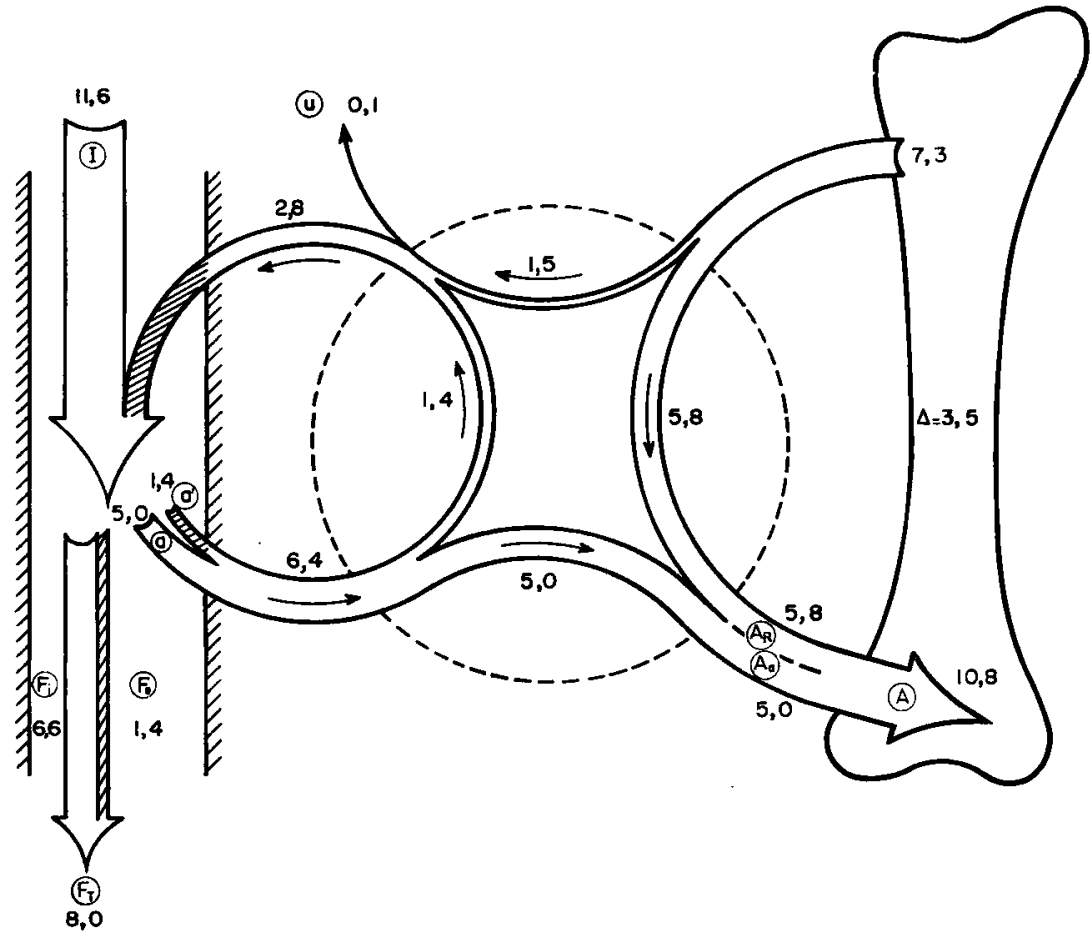

Fig. 2. - Principales voies du métabolisme calcique chez le Porc en croissance (en g de Ca par jour)

$$
\begin{aligned}
\mathbf{I} & =\mathrm{Ca} \text { ingéré } \\
\mathrm{F}_{\mathrm{t}} & =\mathrm{Ca} \text { fécal total } \\
\mathrm{F}_{\mathfrak{1}} & =\mathrm{Cafécal} \text { exogène } \\
\mathrm{F}_{\mathrm{e}} & =\mathrm{Ca} \text { fécal endogène } \\
\boldsymbol{a} & =\mathrm{Ca} \text { exogène absorbé }
\end{aligned}
$$$$
a^{\prime}=\mathrm{Ca} \text { endogène absorbé }
$$$$
\mathbf{U}=\mathrm{Ca} \text { urinaire }
$$$$
\mathrm{A}=\text { accrétion }
$$$$
A a=\text { accrétion exogène }
$$$$
A_{R}=\text { accrétion endogène }
$$ 


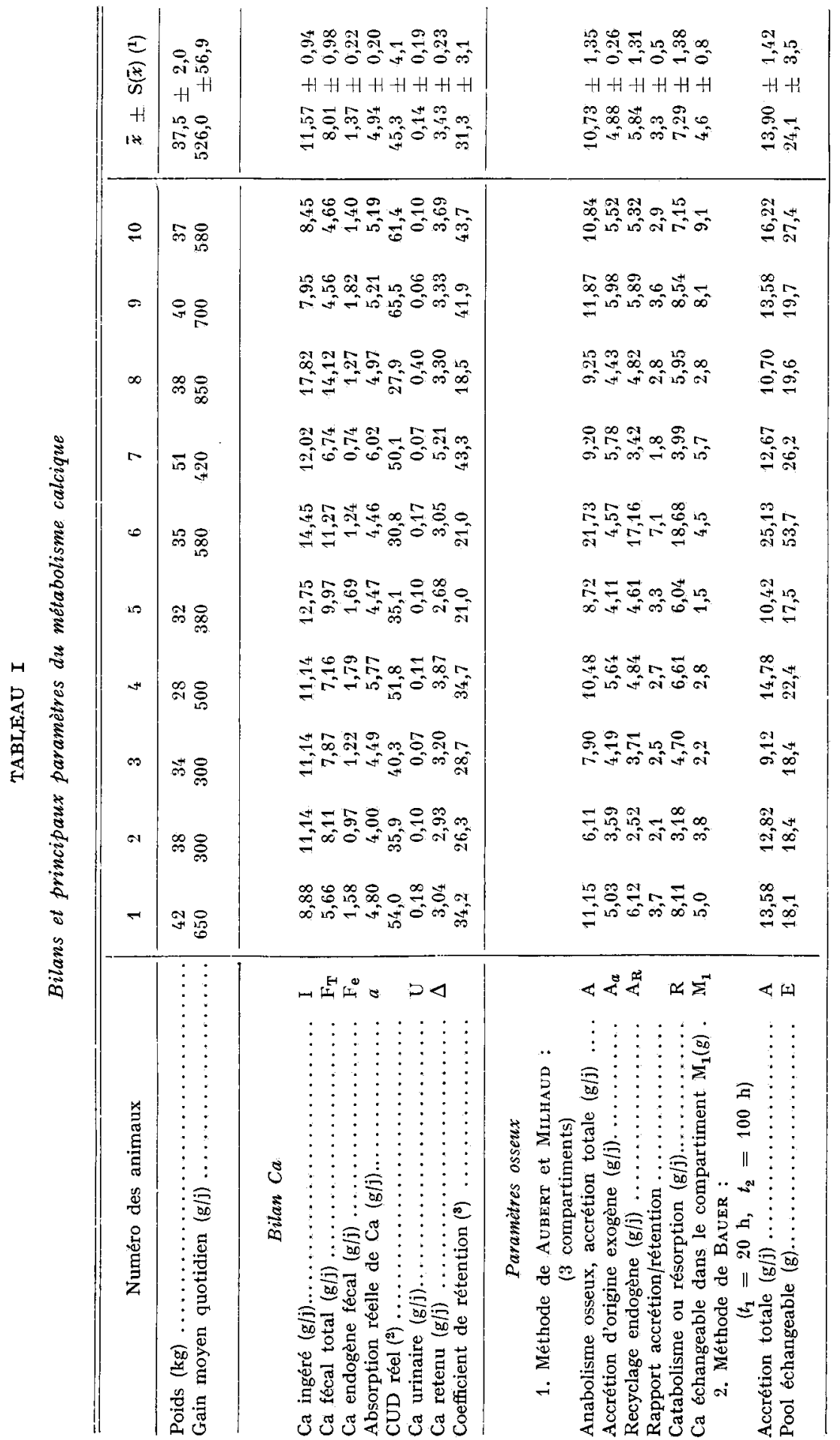




\section{Bilans calciques}

L'ingéré calcique est apparemment l'une des données expérimentales les plus affectées par la variabilité individuelle; il est évident que les animaux supportent plus ou moins bien la contention en cage à métabolisme. Trois d'entre eux (les porcs 2,3 et 4) ont consommé globalement les mêmes quantités d'aliment au cours de la période de mesure de bilans et ont donc ingéré en moyenne la même quantité de Ca.

La calciurie est très faible, c'est pourquoi le coefficient de rétention, qui est de $3 \mathrm{I}, 3 \pm 3$, I p. Ioo de l'ingéré est voisin du coefficient d'utilisation digestive apparente de $32,5 \pm 3,0 \mathrm{p}$. I0o. L'excrétion fécale est pratiquement la plus importante voie d'élimination de calcium endogène : elle représente en moyenne $20 \pm 3 \mathrm{p}$. Ioo de l'excrétion fécale totale de calcium : on a donc un coefficient d'utilisation digestive réelle de $45,3 \pm 4, \mathrm{I}$ p. roo de l'ingéré. On peut aussi calculer l'absorption totale $a+a^{\prime}$ sachant que le calcium endogène sécrété dans l'intestin est en partie réabsorbé ; cela suppose que les sécrétions de calcium d'origine endogène se font, en majeure partie, dans le tractus digestif, plus haut que les principaux sites de l'absorption de $\mathrm{Ca}$, ce qui est généralement admis (MOORE et TYLER, I955; AUBERT et al., I963; NEER et al., I967). Nous avons supposé que 1'utilisation digestive réelle du calcium endogène est la même que celle du calcium exogène d'origine alimentaire.

\section{Aspects osseux}

Les figures 3 et 4 représentent les courbes de décroissance de l'activité spécifique du calcium sanguin après 1 'injection de ${ }^{45} \mathrm{Ca}$, courbes exploitées en vue de déterminer la vitesse d'accrétion du calcium dans l'os. On observe un assez bon accord entre les activités spécifiques du calcium sanguin et urinaire, sauf durant les premières heures après l'injection, et cela confirme les résultats obtenus sur l'Homme par HARRISON et al. (1967) (fig. 4). Dans le tableau 2 sont groupés les coefficients moyens A et $a$, obtenus à partir des décompositions en exponentielles de la forme $\Sigma \mathrm{Ae}-{ }^{a t}$,

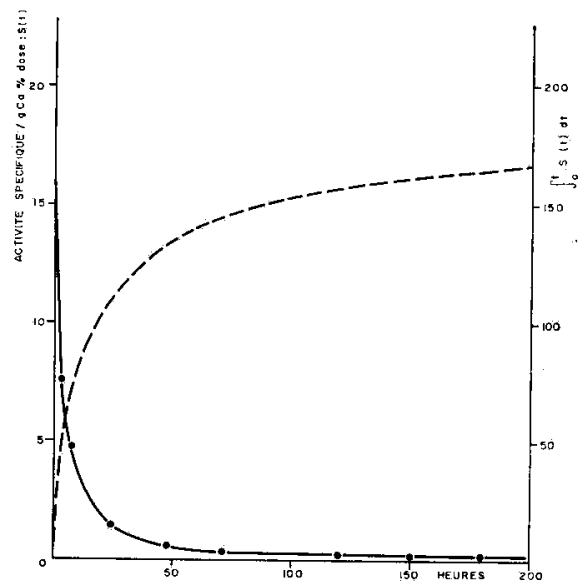

III. 3. - Courbe de décroissance de l'activité spécifique du calcium sanguin (S(t)) et courbe de la fonction intégrale de $\mathrm{S}(t)$ (méthode de BAUER et al.) 
et dont on voit un exemple dans la figure 4 . Les périodes $T_{1} / 2$ de chacune de ces fonctions exponentielles sont respectivement de $I \mathrm{~h} 30,8 \mathrm{~h} 30$ et II $\mathrm{h} 30$. Une exponentielle dont la décroissance serait beaucoup plus rapide et dont il n'a pas été tenu compte, pourrait correspondre à la sortie du ${ }^{45} \mathrm{Ca}$ hors du compartiment sanguin.

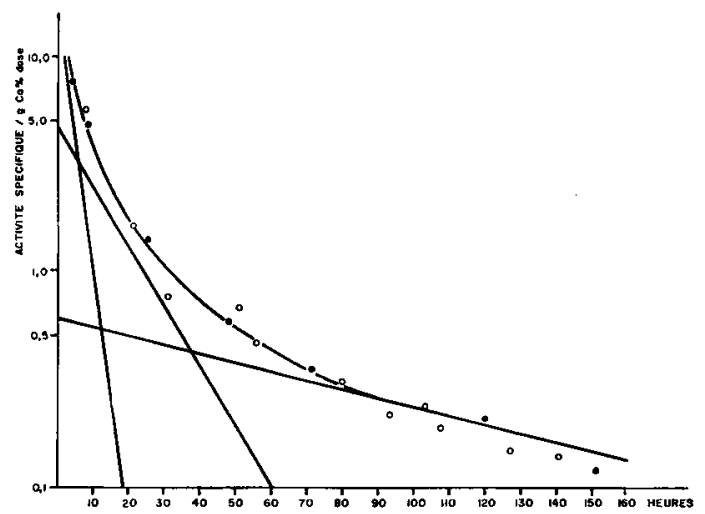

FIG. 4. - Décomposition de la courbe d'activité spécifique du calcium sanguin en trois exponentielles (méthode de AUBERT et Milhaud)

- sang

TABLF:AU 2

Décomposition moyenne des courbes d'activité spécifique du calcium sanguin en trois exponentielles

\begin{tabular}{|c|c|c|}
\hline & A & $a$ \\
\hline 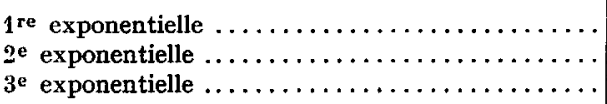 & $\begin{array}{r}24,20 \pm 5,19 \\
4,68 \pm 0,58 \\
0,52 \pm 0,04\end{array}$ & $\begin{array}{l}0,4430 \pm 0,0658 \\
0,0827 \pm 0,0065 \\
0,0060 \pm 0,0009\end{array}$ \\
\hline
\end{tabular}

Équation de la forme $: \mathrm{S}(t)=\mathrm{A}_{1} \mathrm{e}^{-a_{1} t}+\mathrm{A}_{2} \mathrm{e}^{-a_{2} t}+\mathrm{A}_{3} \mathrm{e}^{-a_{3} t}$.

Les valeurs de l'accrétion et des masses de calcium échangeable (tab1. 3) ont été calculées par chacune des méthodes choisies : celle de BAUER, en adoptant deux intervalles de temps différents, soit $20 \mathrm{~h}-50 \mathrm{~h}$, soit $20 \mathrm{~h}-\mathrm{IOO} \mathrm{h}$; celle de AUBERT et Mrlhaud, en tenant compte de 3 exponentielles ou seulement des deux dernières. Dans le cas d'un modèle simplifié à deux compartiments, la vitesse d'échange entre ces compartiments serait de $23,0 \pm 4,5 \mathrm{~g}$ par jour, mais les variations sont très fortes autour de cette moyenne. Connaissant la valeur de l'accrétion totale $A$, on en déduit la valeur de la résorption $\mathrm{R}$ ou mobilisation du calcium osseux par différence entre A et la rétention $\Delta$. Il est également possible d'estimer la part de l'accrétion d'origine endogène $\mathrm{A} a$ en faisant l'hypothèse selon laquelle le pourcentage incorporé à l'os 
de la quantité absorbée (absorption totale $a+a^{\prime}$ ) est égal au pourcentage recyclé de la quantité résorbée de l'os (voir fig. 2), c'est-à-dire : $\frac{\mathrm{A} a}{a+a^{\prime}}=\frac{\mathrm{A}_{\mathbf{R}}}{\mathrm{R}}$. Autrement dit, tout ce qui entre dans le pool calcique a autant de chances d'être incorporé à l'os, quelle que soit son origine, exogène (absorption) ou endogène (résorption).

TABLEAU 3

Comparaison entre différentes méthodes de calcul des principaux paramètres du métabolisme calcique

\begin{tabular}{|c|c|c|}
\hline & $\begin{array}{l}\text { Anabolisme osseux } \\
\text { (Ca } \mathrm{g} / \mathrm{j})\end{array}$ & $\begin{array}{c}\text { Ca échangeable } \\
(\mathrm{g})\end{array}$ \\
\hline$\underset{\text { MÁthode de }}{\text { BAUER et al. }}\left\{\begin{array}{l}t_{1}=20 \mathrm{~h} \\
t_{2}=50 \mathrm{~h} \\
t_{1}=20 \mathrm{~h} \\
t_{2}=100 \mathrm{~h}\end{array}\right\} \ldots \ldots \ldots \ldots \ldots \ldots \ldots \ldots \ldots \ldots \ldots$ & $\begin{array}{l}A=15,58 \pm 1,66 \\
A=13,90 \pm 1,42\end{array}$ & $\begin{array}{l}E=18,5 \pm 2,3 \\
E=24,1 \pm 3,5\end{array}$ \\
\hline $\begin{array}{c}\text { Méthode de } \\
\text { Aubert et Milhaud }\end{array}\left\{\begin{array}{l}2 \text { compartiments............ } \\
3 \text { compartiments........... }\end{array}\right.$ & $\begin{array}{l}V_{0+}=15,62 \pm 2,02 \\
V_{0+}=10,73 \pm 1,35\end{array}$ & $\begin{array}{l}M_{1}=25,5 \pm 6,1 \\
M_{2}=72,4 \pm 14,2 \\
M_{1}=4,6 \pm 0,8\end{array}$ \\
\hline
\end{tabular}

Les valeurs détaillées des principaux paramètres du métabolisme calcique sont groupées dans le tableau $I$, où nous nous sommes limités aux résultats obtenus soit par la méthode de BAUER, avec un intervalle de $20 \mathrm{~h}$-I00 h, soit par la méthode de AUBERT et MIL,HAUD, avec un modèle à 3 compartiments.

La figure 5 est une représentation schématique de la répartition en fonction du temps de la dose de radioactivité administrée. Ces courbes sont obtenues à partir des résultats donnés par la méthode de BAUER. On voit que, dans ce cas, $90 \mathrm{p}$. Ioo de la dose est retenu par l'organisme et que la majeure partie l'est sous forme de ${ }^{45} \mathrm{Ca}$ incorporé par l'accrétion dans 1'os. Après une soixantaine d'heures on peut considérer que les variations ne sont plus très importantes.

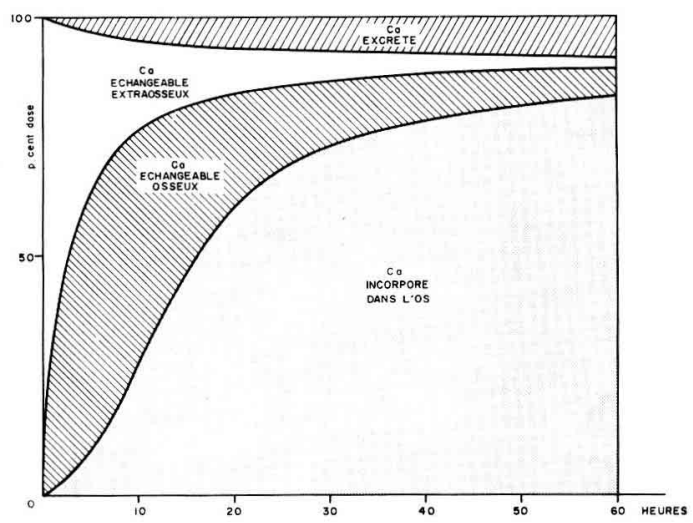

Iig. 5. - Répartition de la dose de radioactivité injectée en fonction du temps 


\section{III. - DISCUSSION}

\section{Les méthodes}

\section{a) Méthode de Aubert et Milmaud.}

Les résultats obtenus dépendent de la précision avec laquelle on détermine la dernière exponentielle, c'est-à-dire la composante la plus lente : les risques d'erreurs sont grands à cause de la dispersion des points et des faibles activités mesurées au bout d'un certain temps. Par ailleurs, la vitesse totale de sortie $V_{T}$ dépend de l'intégrale des fonctions exponentielles. Si l'on néglige une exponentielle, on sous-estime l'intégrale de la courbe de décroissance et l'on surestime la valeur de l'anabolisme osseux : c'est ce qui arrive dans le cas où nous nous limitons à deux exponentielles, c'est-à-dire à deux compartiments (tabl. 3). L'erreur est fonction du rapport des surfaces de chaque exponentielle et, dans le cas présent, l'intégrale de la première exponentielle est importante par rapport aux autres et il convient de ne pas la négliger. Il est donc indispensable de prendre au moins les trois exponentielles que nous avons déterminées. Ire passage de ${ }^{45} \mathrm{Ca}$ du sang vers les autres compartiments doit correspondre à une exponentielle qui a une décroissance très rapide et dont l'intégrale est faible par rapport aux autres. Elle a été négligée dans nos calculs.

\section{b) Méthode de BAuER.}

Dans cette méthode, la principale source d'erreur provient du fait que l'on confond l'activité spécifique du calcium sanguin et l'activité spécifique moyenne du calcium de l'ensemble du pool échangeable de Ca. En fait, le pool se décompose en compartiments, dont le calcium sanguin représente l'une des composantes les plus rapidement échangeables. Une étude antérieure (BESANçon et al., I968), faite sur des porcs en croissance, cherchant à déterminer les limites de validité de la méthode, a montré que l'équilibre entre les différents compartiments du pool ne pouvait être atteint dans les quinze premières heures qui suivent l'injection du radio-élément. Durant les premières heures l'activité spécifique du calcium des compartiments du pool est inférieure à celle du calcium sanguin et augmente jusqu'à lui devenir égale. Ensuite, l'activité spécifique du calcium sanguin est toujours inférieure à celle du calcium des autres compartiments, ce qui entraîne une surestimation de la valeur du pool et une sous-estimation de l'accrétion.

On peut estimer qu'en résolvant l'équation entre $20 \mathrm{~h}$ et $50 \mathrm{~h}$ ou même entre $20 \mathrm{~h}$ et Ioo $\mathrm{h}$ on se place dans des conditions où les écarts entre les différentes activités spécifiques sont faibles, sans toutefois être nuls ; mais les écarts relatifs peuvent être d'autant plus grands qu'on attend plus longtemps, d'où un risque accru de surestimation du pool. Pratiquement, le choix des intervalles 20 heures et 50 ou roo heures montre qu'il n'y a pas de surestimation du pool par rapport aux valeurs obtenues par la méthode de AUBERT et MrLHAUD. Les premiers résultats provisoires que nous avions obtenus sur quatre porcs en croissance (BESANçon et al., I966) donnaient des valeurs un peu sous-estimées du pool et surestimées de l'accrétion, 
parce que la zone de la courbe exploitée pour le calcul de A et E était trop rapprochée du moment de 1'injection du radio-élément.

Il faut remarquer enfin que, d'après NEUMAN et al. (I968), l'équilibre complet entre le calcium circulant et le calcium échangeable osseux ne peut être atteint avant plusieurs dizaines de jours chez le Rat et chez 1'Homme. Il est probable que ce délai est au moins équivalent chez le Porc. Mais y a-t-il jamais un équilibre et, a fortiori, chez l'animal en croissance?...

D'autres sources d'erreurs d'importance moindre viennent de l'estimation de l'intégrale de la fonction $\mathrm{S}(t)$ qui se fait par voie graphique et qui nécessite une extrapolation de la courbe à l'origine. Enfin, les valeurs de la rétention de la dose $\mathrm{R}(t)$ varient peu entre les instants $t_{1}$ et $t_{2}$ choisis et l'erreur expérimentale susceptible d'affecter les valeurs de cette rétention peut être du même ordre de grandeur que la variation de cette donnée entre les deux instants considérés.

\section{Les principales voies du métabolisme calcique}

\section{a) Absorption et rétention calcique}

L'absorption apparente a peu de signification ; elle est équivalente à la rétention lorsque l'excrétion urinaire est négligeable, comme c'est le cas ici. L'absorption réelle, qui représente ce qui est effectivement absorbé à partir du calcium alimentaire, varie assez peu avec le niveau d'apport calcique. C'est l'excrétion fécale qui est l'élément variable, si bien que les coefficients d'utilisation digestive - apparente ou réelle - diminuent fortement lorsque l'ingéré calcique augmente, ce qui est en accord

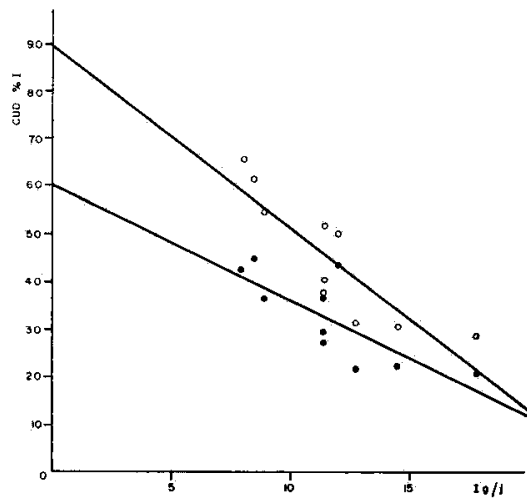

FIG. 6. - Régression entre les coefficients d'utilisation digestive du calcium et le niveau d'apport

- CUD réel.

- CUD apparent

avec des résultats déjà connus (CAUSERET, I954; HANSARd et PlUMLEE, I954; Harrison, I959; Phang et al., I969). Entre les CUD apparents ou réels d'une part et les niveaux d'ingestion de Ca d'autre part, nous avons calculé des coefficients de corrélation qui sont respectivement de $-0,76 \in t-0,87$ (voir fig. 6). Pour des apports calciques élevés les valeurs des CUD apparents et réels se rapprochent, indiquant une moindre proportion de calcium endogène dans les fèces. Il est cependant surpre- 
nant de ne pas observer d'augmentation marquée du calcium endogène fécal, en valeur absolue, contrairement à ce qui a été établi chez le Rat par HANSARD et Plumiee (I954) et chez l'Homme par Phang et al. (I969). De même, l'excrétion urinaire de $\mathrm{Ca}$ augmente assez peu. Ainsi, les mécanismes de l'absorption intestinale sont tels que les quantités de calcium exogène absorbées sont relativement constantes vis-à-vis des variations de l'apport calcique, l'excès de calcium alimentaire non assimilé étant éliminé par les fèces en quantités variables avec le niveau des apports.

L'excrétion fécale de calcium endogène représente cn moyenne $37 \mathrm{mg}$ de $\mathrm{Ca}$ par $\mathrm{kg}$ de poids vif, résultat légèrement supérieur à ceux que HANSARD et al. (I96I) ont trouvé sur des porcs du même âge. Cette excrétion représente la fraction non réabsorbée du calcium endogène des sécrétions digestives. C'est ce qui a été généralement admis dans de nombreuses études sur l'Homme (AUBERT et al., I963; VESIN et Milhaud, I966; NeER et al., I967; Phang et al., I969). Chez le Porc, Moore et TYLER (I955) ont montré que le calcium endogène est sécrété dans le tractus digestif en amont des sites où ce calcium peut être réabsorbé; on admet généralement que ce calcium est bien utilisé. Toutefois, une étude récente de SchEDL et al. (Ig68) a montré, sur le Chien, que le calcium endogène des sécrétions digestives se trouvait dans 1 'intestin en partie sous une forme précipitée non soluble au $\mathrm{pH}$ intestinal, donc non assimilable. Phang et al. (I968) estiment aussi que le calcium endogène n'est pas aussi bien absorbé que le calcium exogène. Ces résultats rendent critiquable l'estimation que nous avons faite de la réabsorption du calcium endogène, qui représente 2I p. Ioo de l'absorption totale de calcium par l'intestin. Il est cependant probable que les écarts entre les CUD du $\mathrm{Ca}$ endogène et exogène ne soient pas très grands et que, par ailleurs, la fraction précipitée de Ca endogène soit faible.

L'existence à peu près certaine d'une réabsorption du calcium endogène et le fait que tout le calcium absorbé ne soit pas utilisé et incorporé dans les tissus calcifiés, nous conduit à considérer un " cycle court " entre le tractus digestif et le sang, dont 1 la vitesse de rotation serait de $I, 3$ à $I, 4 \mathrm{~g}$ par jour (fig. 2). Le cycle complet fait intervenir l'accrétion et la résorption du calcium dont la différence représente la rétention, qui est de $3,4 \mathrm{~g}$ de $\mathrm{Ca}$, soit $3 \mathrm{I}$ p. Ioo de l'ingéré. PECHT (I967) mentionne des valeurs semblables sur des porcs de 30 à $40 \mathrm{~kg}$. HANSARD et al. (I96I) trouvent des résultats un peu supérieurs aux nôtres, mais on ne connaît pas exactement le poids de leurs animaux.

Si l'on rapporte la rétention calcique journalière au poids de l'animal, elle est de $92 \mathrm{mg}$ de Ca par $\mathrm{kg}$ de poids vif. On observe chez le Rat en croissance des valeurs semblables : IOO $\mathrm{mg}$ par $\mathrm{kg}$ de poids vif (HENRY et KON, I953; LEMAIRE, I967). Chez 1'Homme, ce paramètre est beaucoup plus faible : Io $\mathrm{mg}$ par $\mathrm{kg}$ de poids vif chez l'enfant de 3 à I 3 ans (ShERMAN et HAwLEY, I922), moins de I mg par kg de poids vif, chez le jeune adulte (AUBERT et MiLHAUD, I960). Il serait intéressant de rapporter les valeurs de la rétention à la vitesse de croissance ; cela n'est malheureusement pas possible, faute de termes de comparaison suffisamment nombreux. Lc Porc qui, par sa taille, se rapprocherait le plus de l'Homme, a une rétention calcique comparable à celle du Rat si on la rapporte au poids vif. On verra que l'examen des paramètres dynamiques du métabolisme minéral osseux permettra de mieux situcr le Porc parmi les autres espèces. 


\section{b) Le métabolisme minéral osseux.}

Dans une étude de longue durée sur trois porcs, pris entre les âges de 5 mois et 2 ans, Vemmer et al. (Ig66) ont estimé que la demi-vie biologique du ${ }^{45} \mathrm{Ca}$ injecté dans l'organisme était de l'ordre de 500 jours. Cela voudrait dire que les réserves osseuses calciques (dans lesquelles ont été incorporées les traces de radiocalcium) sont en renouvellement et que la moitié $\mathrm{du}{ }^{45} \mathrm{Ca}$ a disparu durant ce temps. En fait, d'après KRAwIEliTZKI et NEHRING (I968), la période d'élimination d'un radionuclide ostéotrope n'est pas constante et présente plusieurs composantes. Cela montre que les phénomènes de résorption osseuse et d'accrétion sont complexes et sont la résultante de plusieurs mécanismes.

Nous avons voulu proposer dans la figure 2 une représentation schématique et globale des principales voies du métabolisme calcique. Quelle est la signification de l'accrétion dans un tel schéma? Deux conceptions opposées s'affrontent : dans le modèle de BAUER, l'accrétion est un mécanisme par lequel le calcium entre dans l'os sous une forme non échangeable ; à 1'opposé, HEANEY et WHEDON (I958) considèrent des échanges qui se font avec des vitesses très différentes, l'accrétion ne représentant dans ce cas que les échanges les plus lents. Les modèles proposés sont ainsi très différents les uns des autres. On serait tenté de chercher à identifier les paramètres du métabolisme minéral osseux, tels que l'accrétion, aux mécanismes définis in vitro. CoHN et al. (1963) distinguent d'une part l'accrétion par apposition, dans laquelle le calcium et le strontium se comportent de la même façon, d'autre part des mécanismes de cristallisation, dans lesquels il y aurait une légère discrimination entre ces deux ostéotropes. TERMINE et POSNER (I967) ont montré que le calcium est d'abord incorporé dans la phase minérale de l'os sous forme amorphe, avant d'être intégré dans la phase cristalline formée par les cristaux d'hydroxyapatite. Enfin, Neuman et Neuman (I958) ont défini in vitro de nombreuses réactions parmi lesquelles des échanges de surface, des échanges intracristallins, des recristallisations, la formation et la croissance de cristaux d'hydroxyapàtite. L'accrétion pourrait correspondre globalement à la formation et à la croissance des cristaux osseux.

L'accrétion que nous mesurons in vivo par des méthodes radio-isotopiques est l'ensemble des mécanismes d'incorporation de calcium dans l'os, autres que les réactions d'échanges rapides; elle inclue les recyclages du calcium libéré par ostéolyse, mis ainsi en circulation dans les fluides corporels ou osseux et réincorporé dans l'os : cela représente $78 \mathrm{p}$. Ioo de la résorption totale et constitue ce que nous avons appelé l'accrétion d'origine endogène. Par hypothèse, nous avons admis que la même proportion, soit $78 \mathrm{p}$. roo du calcium absorbé, est déposé dans l'os et constitue l'accrétion d'origine exogène.

L'accrétion d'origine endogène est l'une des étapes d'un cycle court interne entraînant chaque jour $5,8 \mathrm{~g}$ de calcium; elle a peu de rapports avec la rétention. En revanche, l'accrétion d'origine exogène est directement liée à la rétention par un coefficient de corrélation de $+0,66$.

Les coefficients de corrélation entre l'accrétion exogène $(\mathrm{A} a)$ et l'absorbé réel d'origine alimentaire $(a)$ d'une part, ainsi qu'entre la fraction de calcium mobilisé de l'os et non recyclé $\left(\mathbf{R}_{\ell x}\right)$ et l'excrétion fécale de calcium endogène $\left(\mathrm{F}_{e}\right)$ d'autre part, sont respectivement de $+0,88$ et 0,86 (voir fig. 7 et 8). Si on fait abstraction des deux cycles courts précédemment décrits, on peut concevoir le cycle complet 
suivant : absorption de $\mathrm{Ca}$ exogène - accrétion exogène - résorption ( $\mathrm{Ca}$ non recyclé) - excrétion de Ca endogène. Dans ce cycle, la rétention est bien la différence entre l'accrétion et la résorption. Ce schéma est probablement une simplification un peu abusive et n'a qu'une valeur statistique. L'examen des droites de régression représentées dans les figures 7 et 8 montre que, si l'accrétion exogène était nulle, le peu de calcium absorbé d'origine alimentaire serait engagé dans ce que nous avons appelé le cycle court intestin-sang, et que, si la résorption était nulle, il y aurait encore une excrétion fécale de calcium endogène.

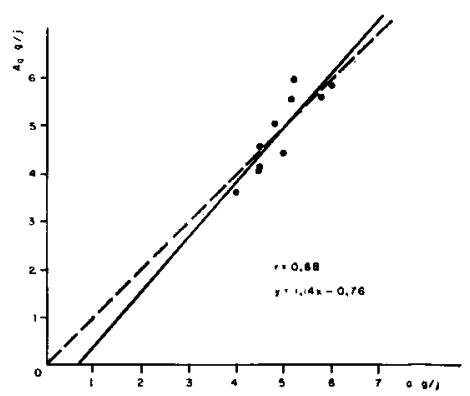

FIG. 7. - Regression entre l'accrétion exogène (Aa) et l'absorption du calcium exogène (a)

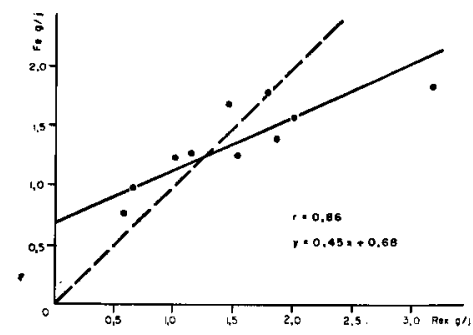

FIG. 8. - Régression entre les quantités de calcium endogène excrétées par voie fécale $\left(\mathrm{F}_{\mathrm{e}}\right)$ et la fraction du calcium mobilise de l'os et non recycle ( $\left.\mathrm{R}_{\mathrm{ex}}\right)$

Un intérêt de la distinction entre l'origine exogène ou endogène de l'accrétion au niveau de l'os serait la possibilité de définir deux caractéristiques osseuses :

- l'accrétion exogène, liée à la rétention calcique, traduirait l'aptitude du squelette à fixer le calcium;

- l'accrétion endogène traduirait en outre l'aptitude de l'os à se renouveler.

c) Le calcium échangeable.

Il est probable que les recyclages au niveau de l'os ne sont pas des réactions d'échange; ces dernières mettent en jeu des quantités de calcium beaucoup plus importantes. Dans un modèle simplifié à deux compartiments, les vitesses d'échanges sont de $23 \mathrm{~g} /$ jour en moyenne et s'élèvent jusqu'à $58 \mathrm{~g} /$ jour pour le porc no $\mathrm{n}^{0}$.

On observe une excellente corrélation de $+0,93$ entre les valeurs de l'accrétion et du pool échangeable ( ${ }^{1}$ ), soit la même valeur que HEANEY et WHEDon (1958) ont

(1) Accrétion et pool échangeable mesurés par la méthode de BAUER entre $20 \mathrm{~h}$ et roo $\mathrm{h}$. 
obtenue sur 1'Homme. On confirme ainsi la liaison qui existe entre les quantités de calcium échangeable et l'activité métabolique de l'os.

Cependant, le pool échangeable mesuré par la méthode de BAUER est une grandeur assez arbitraire et ne correspond pas au fonds commun calculé par la méthode de AUBERT et MILFAUd ; en effet, les compartiments $M_{1}, M_{2}, M_{3}$ sont certainement plus importants. Dans le cas du modèle simplifié à deux compartiments, la somme des masses de calcium dans ces deux derniers dépasse largement la masse du pool échangeable calculée par la méthode de BAUER (tabl. 3). Dans le cas d'un modèle à trois compartiments, $\mathrm{M}_{1}$ représente la masse de calcium des fluides extracellulaires : la valeur obtenue correspond aux chiffres proposés par OSLAGE (r964) pour le calcium extraosseux chez le Porc. Cependant, il est hasardeux de vouloir systématiquement identifier chacun des compartiments du fonds commun calcique à des compartiments corporels anatomiques ou physiologiques bien définis (LARVOR et BARLET, I968).

Les grandeurs du pool échangeable $\mathrm{E}$ que nous avons mesurées n'ont donc qu'une valeur relative.

\section{Conclusions}

Nous avons déjà essayé de situer le Porc parmi d'autres espèces en comparant les valeurs de la rétention rapportée au format. $\mathrm{Si}$, de même, on calcule 1'accrétion par $\mathrm{kg}$ de poids vif on constate que le Porc, avec $290 \mathrm{mg}$ de $\mathrm{Ca}$ par $\mathrm{kg}$ de poids vif, est en position intermédiaire entre l'Homme $\epsilon t$ le Rat et se place à égalité avec le Veau en croissance de $50 \mathrm{~kg}$ (LARVor et BARLET, I968).

On pourrait définir un indice métabolique de la vitalité de l'os qui serait le rapport de l'accrétion sur la rétention; comme le Rat en croissance, le Porc incorpore chaqua jour dans son squelette trois à quatre fois plus de calcium qu'il n'en retient effectivement. Ce mode d'expression est critiquable et ne peut servir à comparer que des animaux pour lesquels les rétentions seraient équivalentes. En effet, chez l'adulte par exemple, la rétention devient très faible ou nulle tandis que l'accrétion demeure importante.

Nous avons vu que la plus grande partie du calcium libéré de l'os par résorption est recyclée et réincorporée : un renouvellement rapide est-il souhaitable? Dans la mesure où l'os ne se déminéralise pas, on peut concevoir que plus le renouvellement minéral osseux sera intense, mieux l'os pourra s'adapter au cours de la croissance à ses nouvelles fonctions de soutien et de réserve minérale. La résorption varie en fonction inverse du niveau des apports calciques, mais le coefficient de corrélation entre ces deux termes est peu élevé : - 0,42. Corn et al. (rg68) ont montré que, chez le Rat soumis à des niveaux calciques très élevés, l'accrétion n'était pas modifiée mais la résorption était fortement diminuée. Récemment, Phang et al. (1969) ont confirmé ce genre de régulation chez 1'Homme. La calcitonine et la parathormone sont certainement responsables de cette action sur le catabolisme osseux. Ainsi, n'y aurait-il pas lieu de penser que des régimes hypercalciques seraient néfastes à un bon métabolisme minéral osseux, mettant en quelque sorte l'os en sommeil, même s'ils favorisent une forte minéralisation? Cette hypothèse conduirait à repenser la notion des besoins calciques de l'animal. On sait quels sont les besoins minima susceptibles d'assurer une bonne croissance et une densité satisfaisante de l'os, mais il est beaucoup plus difficile de situer les besoins calciques optima. Ce besoin pourrait être défini comme étant l'apport alimentaire assurant le meilleur compromis entre 
l'efficacité de l'utilisation par l'animal du calcium ingéré (qui diminue avec les apports), le degré de minéralisation de l'os (qui augmente avec les apports) et les caractéristiques dynamiques iđéales de l'os, qui restent elles-même à définir.

Reçu pour publication en juillet 1969.

\section{SUMMARY}

THE MAIN ROUTES OF CALCIUM METABOLISM IN GROWING PIGS

The main parameters of calcium metabolism were measured in Io growing pigs weighing 30 to $40 \mathrm{~kg}$ after a single intravenous injection of 2 to $3 \mathrm{mCi}{ }^{45} \mathrm{Ca}$.

Calcium balance was estimated, endogenous loss in faeces being measured both by the classical method of isotope dilution and with the aid of cumulative excretion of radioactivity in fæces. The interpretation of the curve of disappearance of specific activity of blood calcium allowed the estimation of the bone parameters by means of the method of BAUER et al., and the method of AUBERT and MilHaud. After comparison and discussion of these two methods a simplified model with three compartments was chosen. The limits of validity of the method of BAUER were defined; this method results in a systematic over-estimation of the rate of incorporation of calcium into bone.

The true digestive utilisation of calcium in the feed was 45 per cent. Loss of endogenous calcium in fæces represented 17 per cent of the total loss of calcium in fæces while excretion in urine was negligible.

The pigs, whose daily gain in weight was 300 to $600 \mathrm{~g}$, retained $3.4 \mathrm{~g}$ calcium per day, that is $3 \mathrm{I}$ per cent of the calcium ingested. Total accretion of calcium, which measures bone anabolism, rise to $10.7 \mathrm{~g}$ per day, and resorption (bone catabolism) was $7.3 \mathrm{~g}$ calcium per day. Nearly half the accretion recorded, that is 46 per cent, was of endogenous origin, as a result of the internal recycling of bone calcium. It is thus possible to measure the capacity of bone to renew itself.

The total of the exchangeable calcium pool was estimated at an average of $24 \mathrm{~g}$.

For its intensity of metabolism of bone mineral the pig resembles the rat rather than man.

\section{RÉFÉRENCES BIBLIOGRAPHIQUES}

Aubert J. P., Milhaud G., I96o. Méthode de mesure des principales voies du métabolisme calcique chez l'Homme. Biochim. Biophys. Acta, 39, 122-139.

Aubert J. P., Bronner F., Richelle L. J., r963. Quantitation of calcium' metabolism theory. J. Clin. Invest., 42, 885-897.

Bauer G. C. H., Carlsson A., Lindquist B., I955 a. Evaluation of accretion, resorption and exchange reactions in the skeleton. Kgl. Fysiograf. Sällskap. Lund. Förh., 25, 3-18.

Bauer G. C. H., Carlsson A., Lindouist B., I955 b. Some properties of the exchangeable bone calcium. Acta physiol. Scand., 35, 67-72.

Bauer G. C. H., Carlsson A., Linsquist B., r96r. Metabolism and homeostatic function of bone, in Comar C. L., Bronner F, Mineral Metabolism, vol. I B, 6og-676, Academic Press, New York, Londres.

Besançon P., Gueguen L., RÉrat A., 1966. Échanges et vitesse d'incorporation du calcium dans le squelette du Porc en croissance. C. R. Acad. Sci. Paris, 263, Ir34-I 137.

Besançon P., Rtuy J., Gutguen L., rg68. Contribution à l'étude du métabolisme calcique au niveau du squelette chez le Porc en croissance par une méthode simplifiée d'exploration cinétique. $C . R$. Acad. Sci. Paris, 267, I754-I757.

Boнr A., I968. On the calcium metabolism and growth rate of rats. Calc. Tiss. Res., 2, suppl., 68.

Bronner F., AUBert J. P., 1965. Bone metabolism and regulation of the blood calcium level in Rats. Amer. J. Physiol., 209, 887-89o.

Causeret J., I954. Recherches sur l'utilisation physiologique du calcium au cours de la croissance chez le Rat. Nouveaux aspects du rôle biologique de la vitamine D. Ann. Zootech., 3, 27I-336.

Cohen Y., Srochet J. C., Ig62. Influence de la croissance sur les échanges calciques étudiée par le calcium-45 chez le Rat. C. R. Acad. Sci. Paris, 254, 392 I-3923. 
Cohn S. H., Lippincotr S. W., Gusmano E. A., Robertson J. S., ig63. Comparative kinetics of ${ }^{47} \mathrm{Ca}$ and ${ }^{85} \mathrm{Sr}$ in man. Radiat. Res., 19, 104-119.

Cohn S. H., Teree, T. M., Gusmano E. A., r968. Effect of varying calcium intake on the parameters of calcium metabolism in the rat. J. Nutrit., 94, 26I-267.

Dallemagne M. J., Bodson P., Fabry C., 1955. Le calcium échangeable de la substance minérale de l'os étudiée à l'aide de ${ }^{45} \mathrm{Ca}$. Biochim. Biophys. Acta, 18, 394-406.

Falkenheim M., UNDerwood E. E., HodGe H. C., I95r. Calcium exchange : the mecanism of adsorption by bone of ${ }^{45} \mathrm{Ca}$. J. Biol. Chem., 188, 805-8r7.

Gutguen L., Rombauts P., I96r. Dosage du sodium, du potassium, du calcium et du magnésium par spectrophotométrie de flamme dans les aliments, le lait let les excreta. Ann. Biol. anim. Bioch. Biophys., 1, 80-97.

Hansard S. L., Plumlee M. P., 1954. Effects of dietary calcium and phosphorus levels upon the physiological behaviour of calcium and phosphorus in the rat. J.Nutr., 54, 17-31.

Hansard S. L., Lyke W. A., Crowder H. M., I96r. Absorption, excretion and utilization of calcium by swine. J.anim. Sci, 20, 292-296.

Harrison H. E., 1959. Factors influencing calcium absorption. Feder. Proc., 18, 1085-1092.

Harrison J., Hitchman A. J. W., Finlay J. M., r967. Calcium determinations in kinetics studies : II. Blood and urine specific activity determinations. Clin. Chem., 13, 760-768.

Heaney R. H., Whedon G. D., 1958, Radiocalcium studies of bone formation rate in human metabolic disease. J. Clin. Endocrinol. Metab., 18, 1246-1267.

HENRY K. M., KoN S. K., I953. The relationship between calcium retention and body stores of calcium in the Rat: effect of age and of vitamine D. Brit. J. Nutri., 7, 147-159.

KRAwielitzKi K., NEhring K., r968. Aufstellung eines Multikompartment-systems für den Stoffwechsel der eines bone-seeking-Elemente. 2. Deutung der einzelnen Kompartments und ergänzende in vitroVersuche. Arch. Tierernähr., 18, 378-39r.

LARvor P., BARLet J. P., I968. Quelques aspects récents du métabolisme calcique : application à la physiologie et à la pathologie des ruminants. Ann. Nutr. Aliment., 22, 155-19o.

Lemaire R., I967. Modifications du métabolisme calcique avec l'âge. C. R. Soc. Biol., 161, $726-729$.

LOFGreen G. P., KLeiber M., I954. Further studies on the availability of phosphorus in alfalfa hay. J. Anim. Sci., 18, 258-264.

LofGreen G. P., r960. The availability of the phosphorus in dicalcium phosphate, bone meal, soft phosphate and calcium phy tate for mature wethers. J. Nutr., 70, 58-62.

Michavo G., Cherian A. G., Motkhtar M., ig63. Calcium metabolism in the Rat studied with ${ }^{45}$ Ca. Effect of age. Proc. Soc. Exp. Biol., 114, 382-384.

Moore J. H., TYLER C., 1955. Studies on the intestinal absorption and excretion of calcium and phosphorus in the pig. 2. The intestinal absorption and excretion of radioactive calcium and phosphorus. Brit. J. Nutr., 8, 8I-93.

Neer R., Berman M., Fisher L., Rosenberg L. E., I967. Multicompartmental analysis of calcium kinetics in normal adult males. $J$. Clin. Invest., 46, I364-1379.

Neuman W. F., Neuman N. W., 1958. The chemical dynamics of bone mineral. The University of Chicago Press, Chicago.

Neuman W. F., Terepka A. R., Canas F., Triffit J. T., r968. The cycling concept of exchange in bone. Calc. Tiss. Res., 2, 262-270.

OSLAGE J., I964. Untersuchungen über die Körperzusammensetzung und den Stoffansatz wachsender Mastschweine. $Z$. Tierphys. Tierernähr. Futterm., 19, 330-357.

Patterson M. S., Greene R. C., 1965. Measurement of low energy beta-emitters in aqueous solution by liquid scintillation counting of emulsions. Anal. Chem., $87,854-857$.

Реснт G., 1967. Wieviel Mineralstoffe brauchen Fleischschweine? Schweinezucht Schweinemast, 15, 198199.

Phang J. M., Berman M., Finerman G. A., Neer R. M., Rosenberg L. E., Hahn T. J., ig69. Dietary perturbation of calcium metabolism in normal man : compartmental analysis. J. Clin. Invest., 48, 67-77.

Schedz H. P., Osbaldiston G. W., Mills T. H., 1968. Absorption, secretion and precipitation of calcium in the small intestine of the dog. J. Physiol., 214, 814-819.

Shermax H. C., Hawley E., I922. Calcium and phosphorus metabolism in Childhood. J. Biol. Chem., 58, 375-399.

Termine J. D., Posner A. S., I967. Amorphous-crystalline interrelationships in bone mineral. Calc. Tiss. Res., 1, 8-23.

Triffitt J. T., Terepka A. R., Neuman W. F., 1968. A comparative study of the exchange in vivo of major constituents of bone mineral. Calc. Tiss. Res., 2, 165-176.

Vemmer H., GütTe J. O., Lenkeit W., I966. Langfristige Untersuchungen über die Retention von parenteral verabreichtem ${ }^{45} \mathrm{Ca}$ an Schweinen. Z. Tierphysiol. Tierernähr. Futterm., 21, 247-253.

Vésin P., Mrlmaud G., r966. Étude de certains aspects actuels du métabolisme calcique. La presse medicale, 74, 1893-1897. 